\title{
RNA interference targeting CHFR enhances taxol chemosensitivity in endometrial cancer cells
}

\author{
XIAOMAO LI ${ }^{*}$, XIAOYUN WANG ${ }^{*}$, YUEBO YANG, CHENGFANG XU and HUIMIN SHEN \\ Department of Obstetrics and Gynecology, The Third Affiliated Hospital, \\ Sun Yat-sen University, 600 Tianhe Road, 510630 Guangzhou, P.R. China
}

Received February 14, 2012; Accepted March 12, 2012

DOI: $10.3892 /$ or.2012.1752

\begin{abstract}
In recent years, it has been reported that CHFR may be a useful biomarker for chemotherapeutic response to microtubule inhibitors in some tumor cells. The purpose of the present study was to test the hypothesis and to elucidate the underlying mechanism in endometrial cancer cells. First, we effectively inhibited CHFR expression at both the mRNA and protein levels using siRNA targeting the CHFR gene in Ishikawa and Hec-la cells. We found that inhibition of CHFR expression significantly enhanced the cytotoxicity of taxol to both cell types, which was confirmed again by colony formation assays. Moreover, suppression of CHFR induced a significant increase of the mitotic index and much lower numbers of cells at the $\mathrm{G}_{2} / \mathrm{M}$ phase in both cells treated with taxol, indicating mitotic checkpoint impairment. On the other hand, the number of apoptotic cells significantly increased in Ishikawa and Hec-la cells transfected with CHFR siRNA after treatment with taxol, which was associated with cyclin B1 nuclear localization. Our data indicate that RNA interference targeting CHFR can sensitize endometrial cancer cells to taxol and CHFR may be a promising molecular target to enhance the therapeutic effect of taxol for endometrial cancer.
\end{abstract}

\section{Introduction}

Endometrial cancer is the most common cancer of the female reproductive tract with 150,000 new cases diagnosed annually worldwide (1). Currently, there is no effective therapy for advanced and recurrent endometrial cancer. Taxol is a cornerstone in the treatment of this malignancy. Unfortunately, the efficacy of taxol is still limited by the development of drug resistance (2). Consequently, further studies that could enhance the therapeutic effect of taxol should be encouraged (3).

Correspondence to: Professor Xiaomao Li, Department of Obstetrics and Gynecology, The Third Affiliated Hospital, Sun Yat-sen University, 510630 Guangzhou, P.R. China

E-mail: tigerlee777@163.com

*Contributed equally

Key words: endometrial cancer cells, CHFR, taxol, RNA interference
A novel mitotic checkpoint protein of significant interest in chemosensitizing activity of taxol is CHFR (checkpoint with forkhead-associated and ring finger), which is localized to chromosome $12 q 24.33$ (4). In cellular response to mitotic stress by microtubule inhibitors, such as taxol, CHFR activation delays chromosome condensation during prophase and increases the ability of cells to survive the stress (5).

Some reports found that CHFR inactivation may play a key role in sensitivity of cancer cells to microtubule inhibitors by affecting mitotic checkpoint function (6-8). Moreover, we previously reported that CHFR suppression regulated by hypermethylation might sensitize endometrial cancer cells to taxol (9). Now we hypothesized that knockdown of CHFR in endometrial cancer cells would make them more sensitive to taxol. To test the hypothesis and elucidated the underlying mechanism, we employed the siRNA technique to silence CHFR expression. Then we determined whether taxol-mediated chemotherapy in combination with suppression of CHFR could be more effective in endometrial cancer cells.

\section{Materials and methods}

Cell culture. The human endometrial cancer cell lines, Ishikawa and Hec-1a, were obtained from American Type Culture Collection (Manassas, VA). All cells were maintained in Dulbecco's modified Eagle's medium (Gibco-BRL, Grand Island, NY) supplemented with $10 \%$ fetal bovine serum, under $5 \% \mathrm{CO}_{2}$ at $37^{\circ} \mathrm{C}$.

Transfection of siRNA. Ishikawa and Hec-la cells were transfected with CHFR siRNA or negative control siRNA (Shanghai GenePharma Co., China) using Lipofectamine 2000 (Invitrogen) according to the manufacturer's directions. The optimal siRNA sequences for CHFR selection were: 5'-CGACAGCAGUCCA GG AUUAdTdT-3' (sense) and 3'-dTdT GCUGUCGUCAGGUC CUAAU-5' (antisense). The negative-control siRNA sequences were: 5'-UUCUCCGAACGUGUCACGUTT-3' (sense) and 5'-ACGUGACACGUUCGGAGAATT-3' (antisense). Blank control (mock) cells were treated with Lipofectamine 2000 but not with siRNA. Cells were harvested $48 \mathrm{~h}$ after siRNA-treatment for the following analysis.

Quantitative real-time PCR. Total RNA from Ishikawa and Hec-1a was extracted with TRIzol reagent (Invitrogen). cDNA 
was then generated from $1.0 \mu \mathrm{g}$ of total RNA with avian myeloblastosis virus reverse transcriptase (Takara, Otsu, Japan). Amplification was carried out with an ABI PRISM 7000 (Applied Biosystems, Tokyo, Japan) using SYBR green reagent for detection according to the protocol of SYBR Premix Ex $\mathrm{Taq}^{\mathrm{TM}}$ kit (Takara). Primer sequences for CHFR were 5'-CCTCA ACAACCTCGTGGAAGCATAC-3' (sense) and 5'-TCCTGGCA TCCATACTTTGCACATC-3' (antisense); primer sequences for $\beta$-actin were 5'-TGGCACCCAGCACAATGAA-3' (sense) and 5'-CTAAGTCATAGTCCGCCTAGAAGCA-3' (antisense). The comparative threshold method was used to calculate the relative amount of mRNA of treated sample in comparison with control samples (10).

Western blot analysis. The cells were harvested, lysed and centrifuged at $15,000 \mathrm{rpm}$ for $5 \mathrm{~min}$ at $4^{\circ} \mathrm{C}$. Protein content in the supernatants was determined by a BCA protein assay kit. Equal amounts of protein were separated by $10 \%$ SDS-PAGE, transferred onto PVDF membranes and then incubated with CHFR (1:500, Santa Cruz, USA), $\beta$-actin (1:2000, Santa Cruz, USA) antibody overnight at $4^{\circ} \mathrm{C}$. After incubation with secondary antibody (KPL, Gaithersburg, MD), membrane was treated with ECL-Western blot detecting reagent (Amersham Biosciences KK, Tokyo, Japan). Protein bands detected were estimated using Quantity One software (Bio-Rad Laboratories, Hercules, CA). The density measurement was correlated to protein expression and normalized to $\beta$-actin.

Cell cytotoxicity assay. The cells (5,000 cells/well) seeded in 96-well plates were transfected with $50 \mathrm{nM}$ siRNA for $48 \mathrm{~h}$. Transfected and untransfected cells were incubated with fresh medium containing various concentrations of taxol for another $24 \mathrm{~h}$. Cell viability was assayed by a tetrazolium salt (WST-8)-based colorimetric assay according to the manufacturer's instructions of the Cell Counting Kit 8 (CCK-8; Dojindo, Kumamoto, Japan). Cytotoxicity was determined as follows: (1 - absorbance value of cells in the taxol-treated group/absorbance value of cells in the control group) $\mathrm{x} 100 \%$.

Colony formation assay. The transfected and untransfected cells were plated at a density of 500 cells/well into 6 well-plates and treated with taxol at a final concentration of $1.0 \mu \mathrm{g} / \mathrm{ml}$ for $24 \mathrm{~h}$. After 14 days, the resultant colonies were stained with crystal violet for $15 \mathrm{~min}$. After washing out the dye, colonies ( $\geq 50$ cells) were counted under the microscope and photographed. Three random fields were counted for each triplicate samples.

Mitotic index. After the above treatment, the cells were fixed in 4\% paraformaldehyde for $15 \mathrm{~min}$ at room temperature, stained with Hoechst 33342 (5 $\mu \mathrm{g} / \mathrm{ml}$; Invitrogen). The mitotic index, which represents the fraction of cells with fully condensed chromosomes, was counted among at least 300 cells under a fluorescence microscope (Nikon TE2000-U, Tokyo, Japan). We took random photos (Olympus BX51, Tokyo, Japan) of eight visual fields using 200-fold magnification and counted the total cell and positive cell numbers to calculate the expression percentage.

Cell cycle assay. The transfected and untransfected cells were plated at a density of $2 \times 10^{5}$ cells/well into 6 well-plates treating with taxol $(1.0 \mu \mathrm{g} / \mathrm{ml})$. Twenty-four hours later, the cells were harvested and cell cycle was analyzed with Cycle Test Plus DNA Reagent Kit (Becton-Dickinson, San Jose, CA) according to the manufacturer's protocol. Cells were washed with PBS and fixed with $70 \%$ ethanol then stained with propidium iodide (PI). The relative proportions of cells in the $G_{0} / G_{1}, S$, and $G_{2} / M$ phases were determined by FACSCalibur ${ }^{\mathrm{TM}}$ flow cytometry (BD Biosciences, San Diego, CA).

Cell apoptosis assay. After the above treatment, the cell apoptosis was analyzed with using the Annexin V-FITC Apoptosis Detection Kit (BD Biosciences) according to the manufacturer's protocol. Briefly, the cells were collected, washed with PBS, suspended in $100 \mu \mathrm{l}$ binding buffer, incubated with $10 \mu \mathrm{l}$ Annexin V-FITC and $5 \mu \mathrm{l}$ of propidium iodide (PI) for $15 \mathrm{~min}$ in the dark, finally $400 \mu \mathrm{l}$ binding buffer was added to cells. The data were acquired immediately with FACSCalibur flow cytometry (BD Biosciences).

Immunofluorescence. After the above treatment, cells were fixed with $4 \%$ paraformaldehy for 20 min following permeabilization with $3 \%$ BSA and $0.1 \%$ Triton X-100 in PBS for 30 min. Cells were subsequently incubated for overnight at $4^{\circ} \mathrm{C}$ with cyclin $\mathrm{B} 1$ antibody (1:500, Santa Cruz). Secondary antibody conjugated to Alexa Fluor 488 (1:200, Molecular Probes) was then used. Nuclei were detected using Hoechst 33342 (5 $\mu \mathrm{g} / \mathrm{ml}$; Invitrogen). Cells were observed under a fluorescence microscope (Nikon TE2000-U).

Statistical analysis. All experiments were performed in triplicate and found to be reproducible. The results were expressed as mean \pm SD. Statistical analysis was performed by one-way ANOVA or Student's t-test. $\mathrm{P}<0.05$ was considered statistically significant.

\section{Results}

Inhibition of CHFR expression by siRNA. To test the silencing effect of siRNA targeting CHFR gene, real-time PCR and western blotting were performed to detect the expression of CHFR mRNA and protein in two endometrial cancer cell lines, Ishikawa and Hec-la. Compared with mock or control-siRNA cells, the relative expression level of CHFR mRNA was reduced by $70 \%$ in Ishikawa CHFR-siRNA cells and $75 \%$ in Hec-1a CHFR-siRNA cells ( $<<0.05$; Fig. 1A). CHFR expression at the protein level was significantly inhibited in both cells, which was consistent with the mRNA level ( $p<0.01$; Fig. 1B). There was no obvious difference between mock and control-siRNA cells $(\mathrm{p}>0.05)$. These results indicated that CHFR expression was significantly suppressed by CHFR siRNA in Ishikawa and Hec-la cells.

Suppression of CHFR sensitized endometrial cancer cells to taxol. Previous studies suggested that CHFR might be involved in chemoresistance to microtubule inhibitors in many cancer types (6). To test this idea, we addressed whether suppression of CHFR could enhance sensitivity of taxol in two endometrial cancer cell lines. Forty-eight hours after transfection of siRNA, cells were exposed to various concentrations of taxol for $24 \mathrm{~h}$. When CHFR expression was decreased in the cells, we observed that the cytotoxicity significantly increased by 

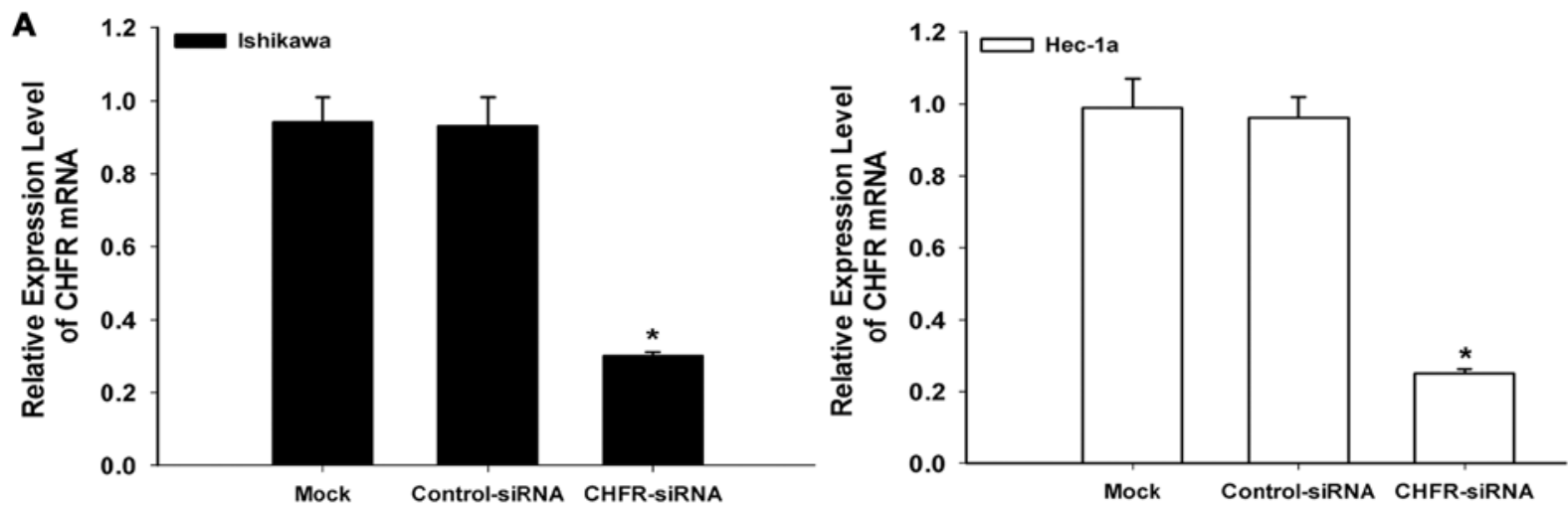

B
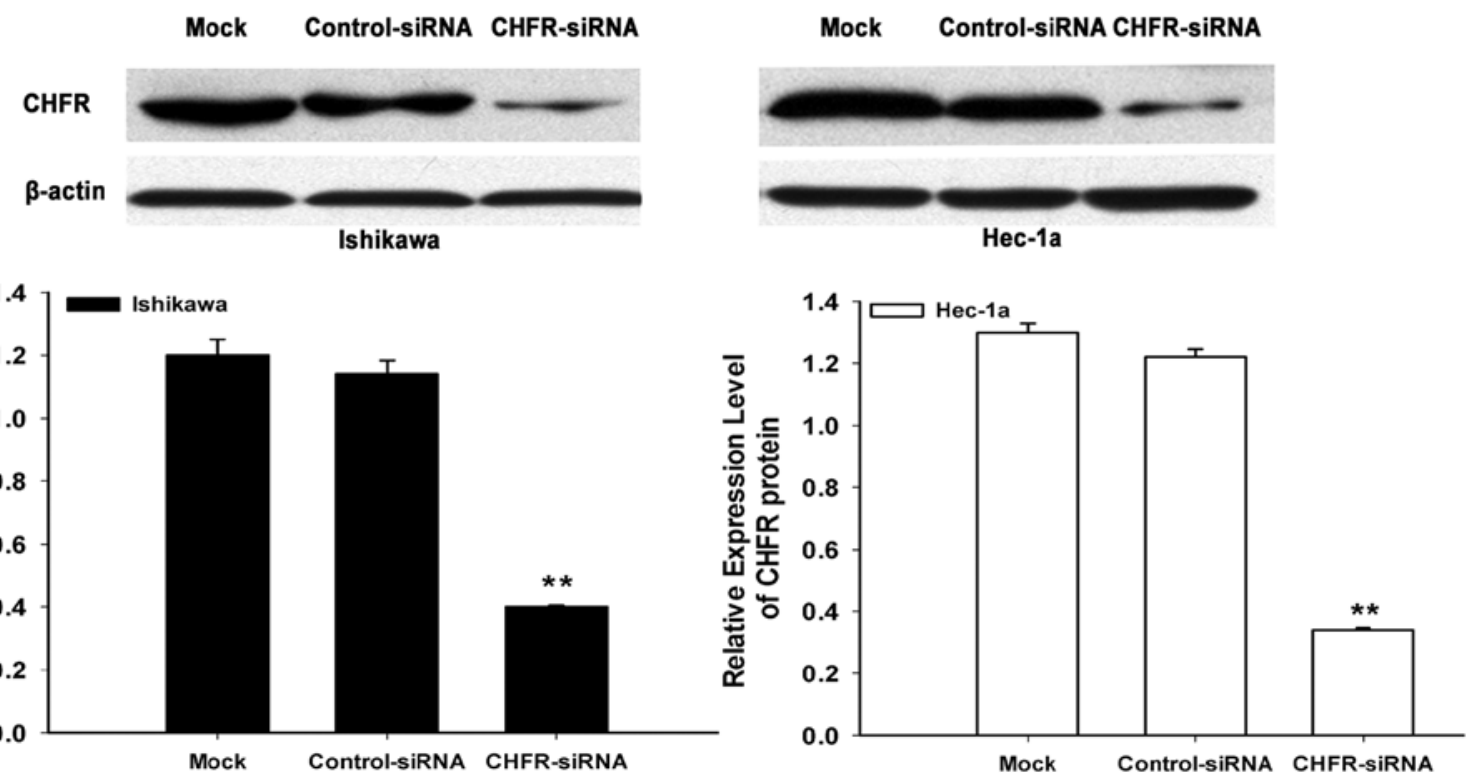

Figure 1. Inhibition of CHFR expression by siRNA in endometrial cancer cells. (A) CHFR and $\beta$-actin mRNA levels were determined by real-time PCR $48 \mathrm{~h}$ after transfection. (B) The silencing effect of CHFR siRNA at the protein level was detected by western blotting $48 \mathrm{~h}$ after transfection. Data are presented as mean $\pm \mathrm{SD}(\mathrm{n}=3) .{ }^{*} \mathrm{p}<0.05 ;{ }^{* *} \mathrm{p}<0.01$.

CHFR siRNA as compared with mock or control siRNA. After treatment with taxol at a final concentration of $1.0 \mu \mathrm{g} / \mathrm{ml}$ for $24 \mathrm{~h}$, cytotoxicity increased to $67 \pm 5.1 \%$ in Ishikawa CHFRsiRNA cells (compared with $27.6 \pm 3 \%$ in control-siRNA cells) and $89.2 \pm 5.7 \%$ in Hec-la CHFR-siRNA cells (compared with $39.4 \pm 2.8 \%$ in control-siRNA cells) $(\mathrm{p}<0.05$; Fig. $2 \mathrm{~A}$ ), indicating a significantly increased sensitivity to taxol by suppression of CHFR expression.

The increased sensitivity of endometrial cancer cells with CHFR suppression to taxol was confirmed again by subsequent colony formation assay. Fourteen days after exposure to taxol (1 $\mu \mathrm{g} / \mathrm{ml})$ for $24 \mathrm{~h}$, both CHFR-siRNA cell types exhibited much smaller colony diameter, and their colony formation numbers were reduced by $80 \%-90 \%$, compared with controls $(\mathrm{p}<0.05$; Fig. 2B).

Knockdown of CHFR induced mitotic checkpoint impairment and entry of cells to mitosis when treated with taxol. Cancer cells lacking CHFR show mitotic checkpoint impairment when treated with microtubule inhibitors (7). We evaluated the impaired checkpoint function in endometrial cancer cells with CHFR suppression after treatment with taxol, using mitotic index. Exposed to taxol $(1 \mu \mathrm{g} / \mathrm{ml})$ for $24 \mathrm{~h}$, the CHFR-siRNA cells (Ishikawa and Hec-1a) showed condensed chromosomes and higher mitotic indexes than control-siRNA cells. Especially in Hec-la CHFR-siRNA cells, the mitotic index was $87 \pm 3.5 \%$, compared with $6 \pm 1.4 \%$ in control-siRNA cells $(\mathrm{p}<0.01$; Fig. $3 \mathrm{~A})$, indicating that knockdown of CHFR led to mitotic checkpoint impairment after treatment with taxol.

As a cell cycle checkpoint gene, CHFR induces cell cycle arrest in $G_{2}$ phase $\left(G_{2}\right.$ arrest) to allow repair of damaged DNA during mitotic stress (8). When Ishikawa and hec-1a cells (expressing CHFR) were incubated for $24 \mathrm{~h}$ with taxol $(1 \mu \mathrm{g}$ / $\mathrm{ml})$, large numbers of cells remained at $\mathrm{G}_{2} / \mathrm{M}$ phase $(30-35 \%)$ with normal checkpoint function. In contrast, both CHFRsiRNA cells (lack CHFR) showed a decrease in the number of cells at $\mathrm{G}_{2} / \mathrm{M}$ phase (6-9\%) after treatment with taxol for $24 \mathrm{~h}$ (Fig. 3B), indicating that due to mitotic checkpoint impairment, CHFR suppression may induce endometrial cancer cells to enter mitosis but not arrest in prophase when treated with taxol.

More apoptosis induced by CHFR suppression was associated with cyclin B1 nuclear localization. In this study, we also determined apoptosis of endometrial cancer cells with CHFR 
A

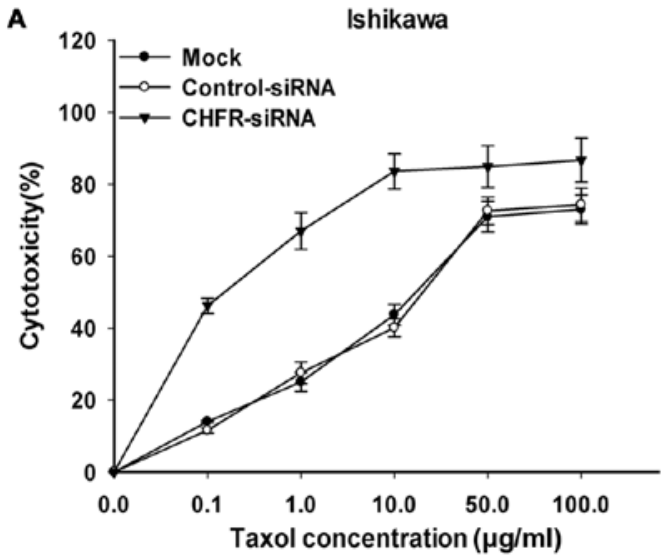

B

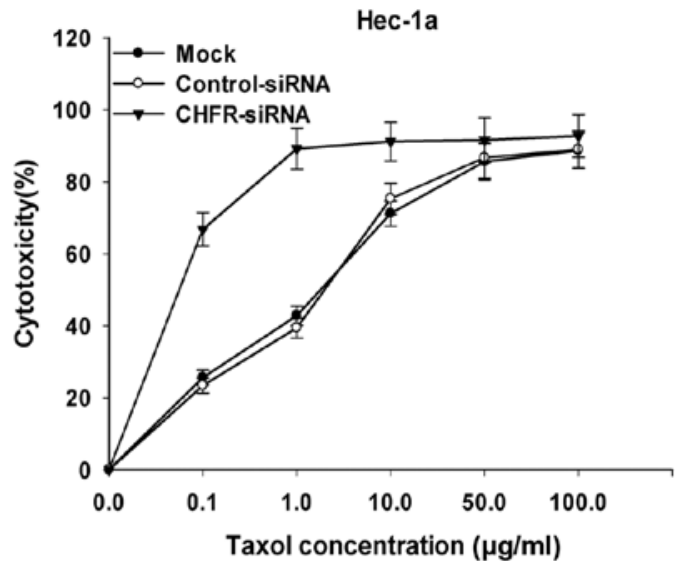

Control-siRNA

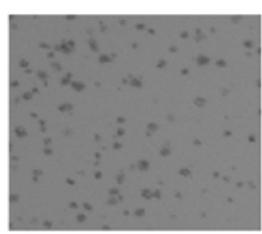

CHFR-SIRNA
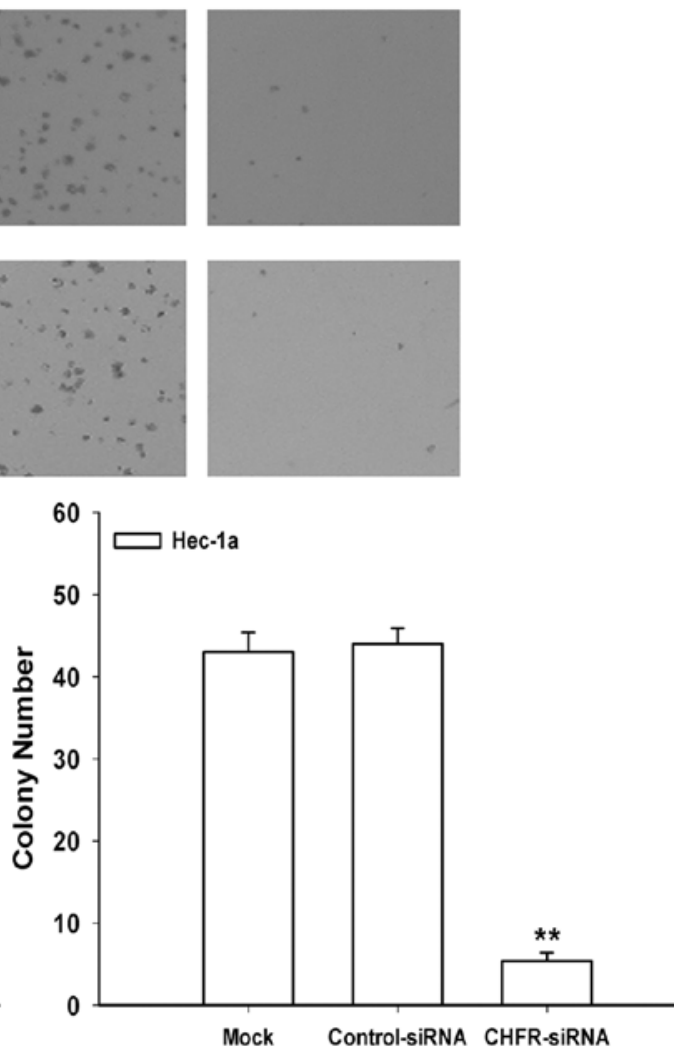

Figure 2. Suppression of CHFR sensitizes endometrial cancer cells to taxol. (A) Transfected cells were exposed to various concentrations of taxol for 24 h, the cytotoxicity was evaluated by CCK-8. Fourteen days after exposure to taxol $(1 \mu \mathrm{g} / \mathrm{ml})$ for $24 \mathrm{~h}$, the CHFR-siRNA cells exhibited much smaller colony diameter, and their colony formation numbers were reduced. Data are presented as mean $\pm \mathrm{SD}(\mathrm{n}=3) .{ }^{*} \mathrm{p}<0.05 ;{ }^{* * *} \mathrm{p}<0.01$.

suppression after treatment with taxol, using flow cytometric analysis. We found that the apoptotic rate was increased from $4.04 \pm 0.87 \%$ in Ishikawa mock cells to $17.65 \pm 1.8 \%$ in Ishikawa CHFR-siRNA cells, and from $6.89 \pm 1 \%$ in Hec-1a mock cells to $20.45 \pm 1.76 \%$ in Hec-1a CHFR-siRNA cells ( $<<0.05$; Fig. 4). It showed that inhibition of CHFR could promote apoptosis of endometrial cancer cells when treated with taxol.

On the other hand, it was reported that cyclin B1 accumulated in the cytoplasm between $S$ and $G_{2}$ phase, and then localized in the nuclei during prophase (11). Localization of cyclin B1 is among the factors determining the cellular decision to undergo apoptosis in response to DNA damage (12). Thus, we examined the distribution of cyclin B1 after treating endometrial cancer cells with taxol. When Ishikawa and Hec-la cells transfected with negative or mock siRNA were treated with taxol, cyclin B1 mainly localized in the cytoplasm. By contrast, in CHFRsiRNA cells treated with taxol, cyclinB1 mainly accumulated in the nuclei (Fig. 5). The results suggest that the greater amount of apoptosis induced by CHFR suppression, which impelled endometrial cancer cells to enter into mitosis without repair of genotoxic damage when treated with taxol, may be correlated with cyclin B1 translocation to the nuclei.

\section{Discussion}

Advanced and recurrent endometrial cancer remain major clinical problems by causing considerable morbidity and mortality in women worldwide. Apart from the standard approaches, novel 
A
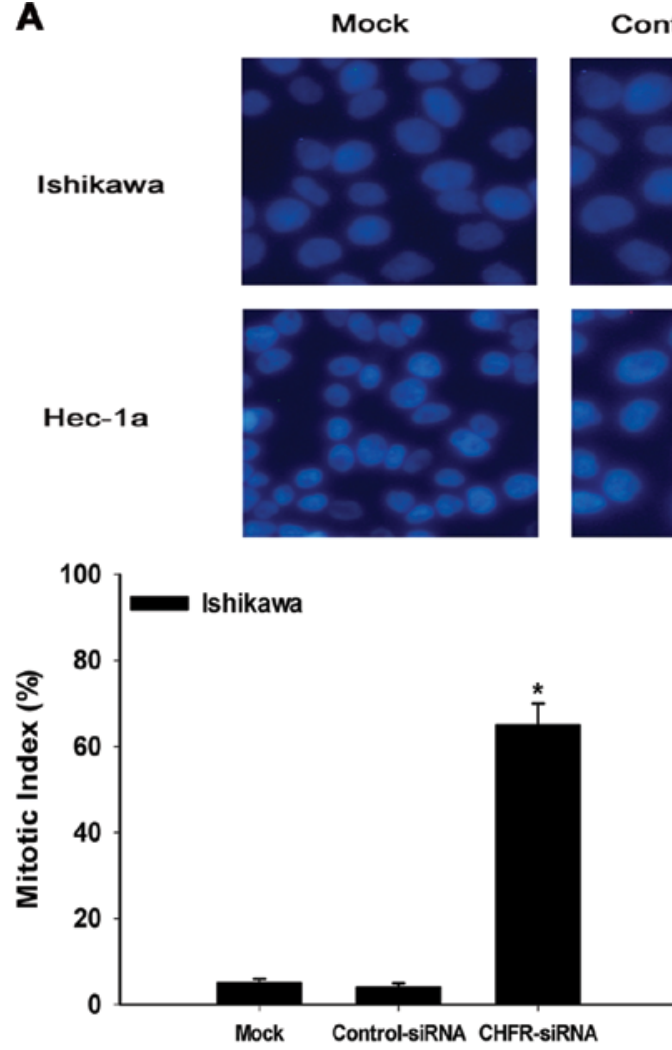

B

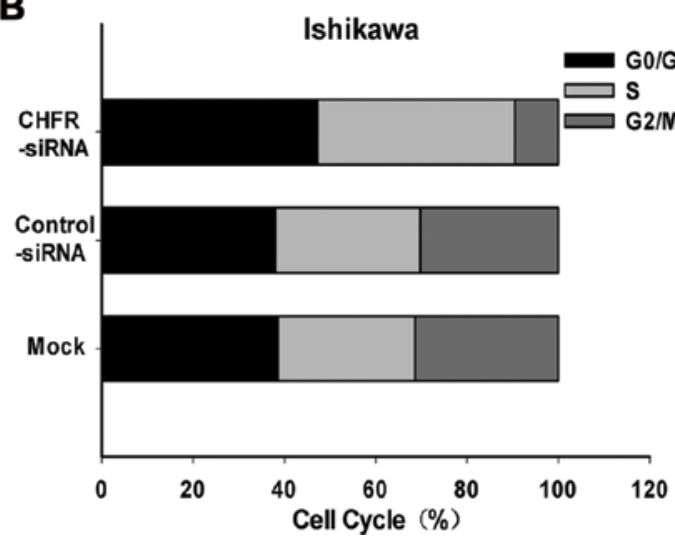

Control-siRNA
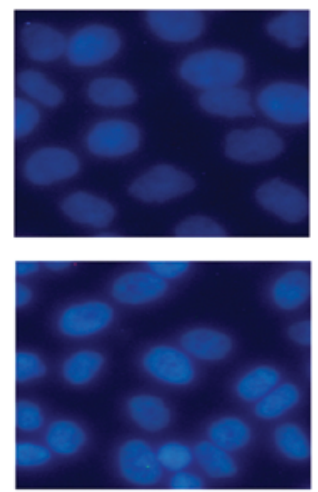

CHFR-siRNA
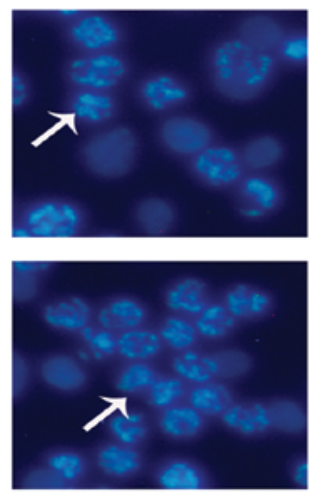
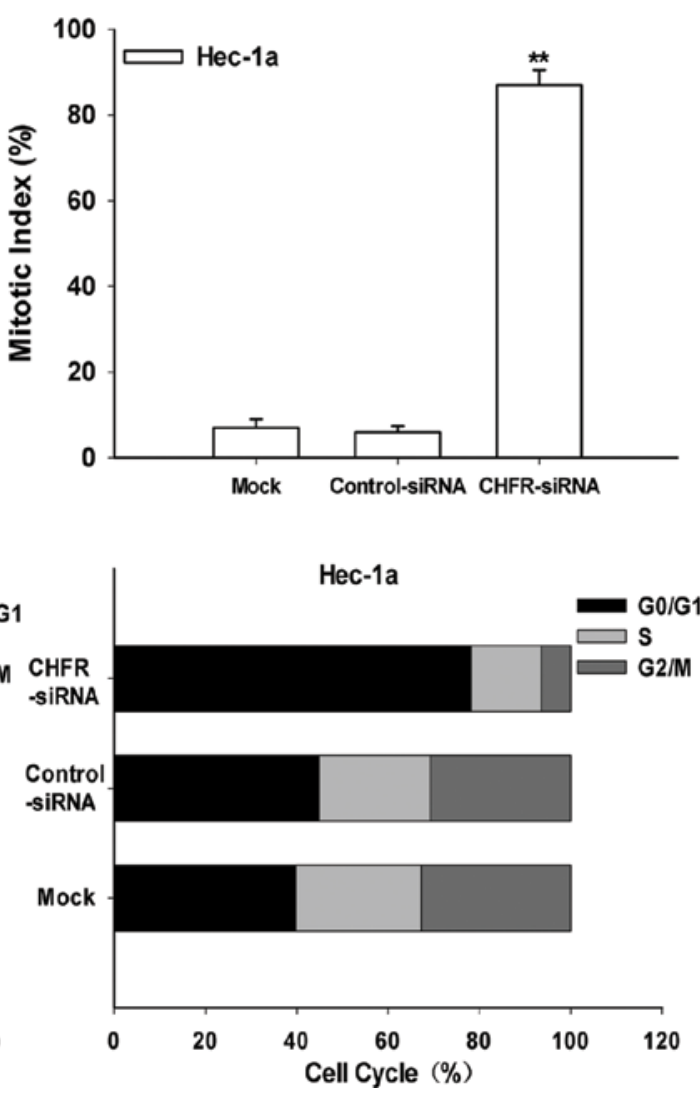

Figure 3. Knockdown of CHFR induces mitotic checkpoint impairment and entry of cells to mitosis when treated with taxol. (A) Transfected cells after treatment of taxol $(1.0 \mu \mathrm{g} / \mathrm{ml})$ for $24 \mathrm{~h}$ were stained with Hoechst-33342 and then nuclei were observed under a fluorescent microscope using a blue filter (x400). White arrows indicate mitotic cells. The mitotic cells showed condensed chromosomes. Data are presented as mean $\pm \mathrm{SD}(\mathrm{n}=3) .{ }^{*} \mathrm{p}<0.05$; ${ }^{* *} \mathrm{p}<0.01$. (B) Incubation for $24 \mathrm{~h}$ with taxol $(1 \mu \mathrm{g} / \mathrm{ml})$, transfected cells were stained with propidium iodide and analyzed by flow cytometry. Data are representative of 3 independent experiments.

potent molecular agents for anticancer therapy are required $(13,14)$. In the past 10 years, CHFR as a mitotic checkpoint has gained more and more attention as a possible novel biomarker for chemotherapeutic response to microtubule inhibitors (taxol) in human cancers (15).

RNA interference is a mechanism of post-transcriptional gene silencing. Small interfering RNA (siRNA) has served as a powerful technology to knock down the expression of target genes (16). Current studies and clinical trials demonstrate that manipulation of the RNAi mechanism by use of targeted siRNA offers a novel and attractive therapeutic option against cancer (17). There are reports showing that silencing of the IKKع gene by siRNA inhibited invasiveness and growth of breast cancer cells (18) and siRNAs targeting the individual CK2 subunits enhanced chemosensitivity to gemcitabine in human pancreatic cancer cells (19). In our study, the siRNA technology was applied to suppress CHFR expression.

Previously, we had demonstrated that CHFR suppression regulated by hypermethylation might sensitize endometrial cancer cells to taxol (9). Among 6 endometrial cancer cell lines, Ishikawa and Hec-1a cells with high CHFR expression were resistant to taxol. Therefore, we chose these two cell lines in our present study.

First, we effectively inhibited CHFR expression by using siRNA targeting CHFR gene in Ishikawa and Hec-la cells. Then, we found that inhibition of CHFR expression significantly enhanced the cytotoxicity of taxol in both endometrial cancer cells. The enhancement of taxol cytotoxicity to Ishikawa and 

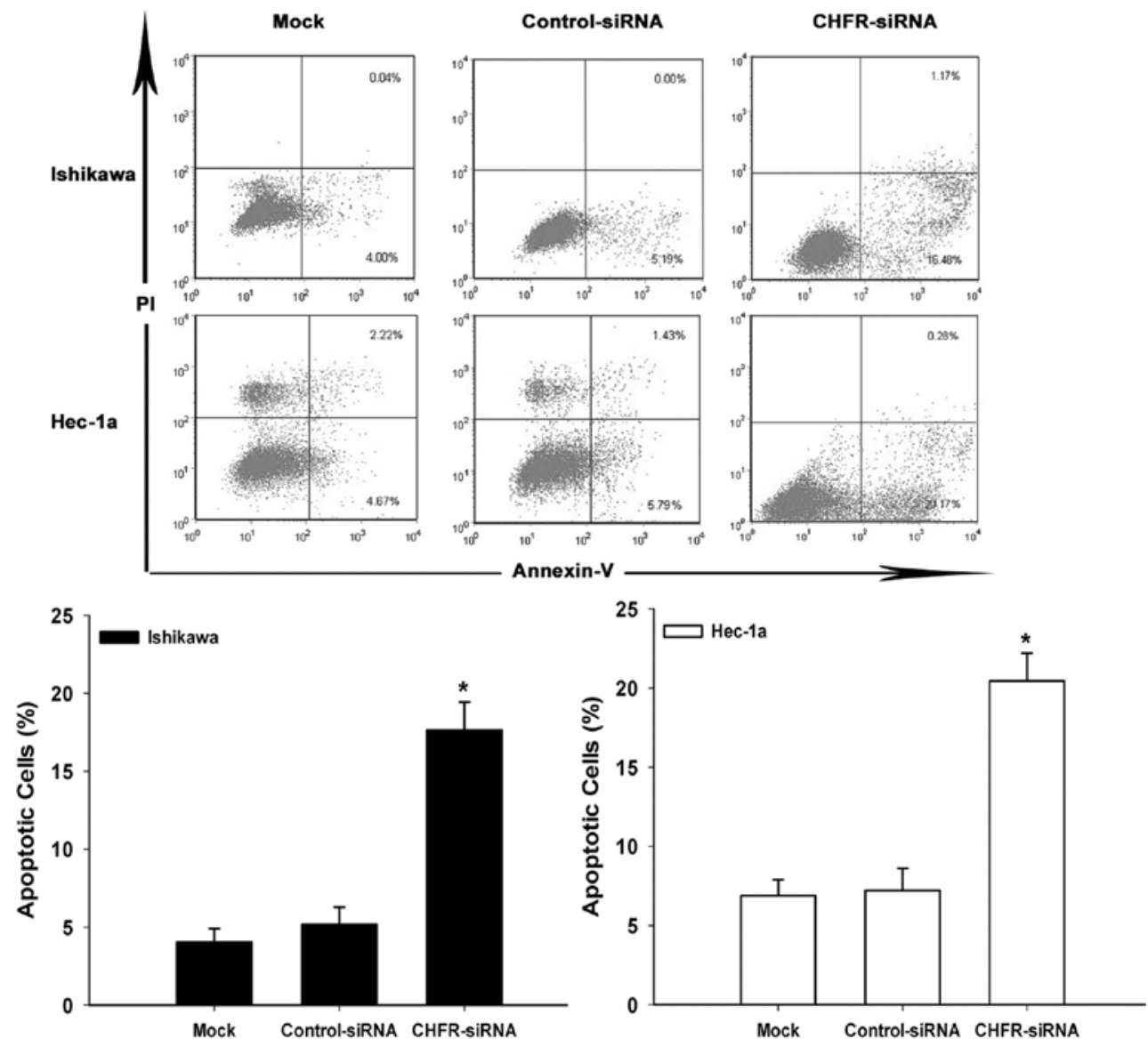

Figure 4. CHFR suppression induced higher numbers of apoptotic cells. Transfected cells after treatment with taxol $(1 \mu \mathrm{g} / \mathrm{ml})$ were stained with Annexin-V+PI and analyzed by flow cytometry. Data are presented as mean $\pm \operatorname{SD}(n=3)$. ${ }^{*} \mathrm{p}<0.05$.
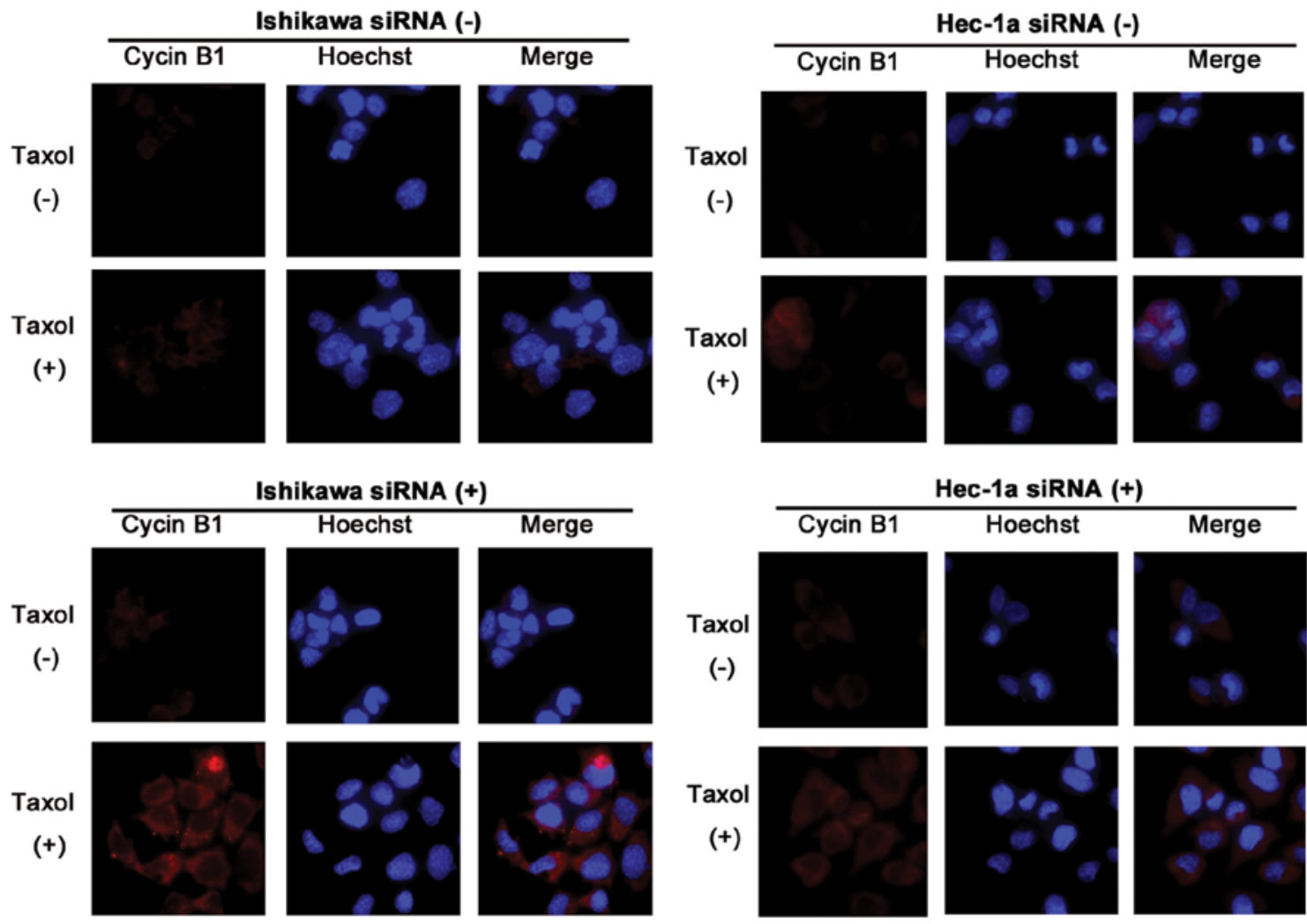

Figure 5. CHFR suppression induces cyclin B1 accumulation in the nuclei during mitotic stress. When CHFR-expressing Ishikawa and Hec-1a cells were treated with taxol, cyclin B1 mainly localized in the cytoplasm. In contrast, treating CHFR-siRNA cells with taxol, cyclinB1 accumulated in the nuclei. The results presented are an average of 6 random microscopic fields from 3 independent experiments. 
Hec-la cells transfected with CHFR siRNA was confirmed again by colony formation assays. The results highlight the potential of utilizing CHFR as a molecular target for cancer therapy.

CHFR regulates an early mitotic checkpoint, during prophase, in response to the disruption of normal microtubule formation or stabilization as assessed after treatment with microtubule inhibitors such as taxol (20). The CHFR-mediated prophase checkpoint is typically monitored by calculating the mitotic index of cells treated with microtubule inhibitors (21). In our study, suppression of CHFR induced condensed chromosomes and an obvious increase of the mitotic index during mitotic stress in Ishikawa and Hec-la cells treated with taxol. Much lower numbers of cells at $\mathrm{G}_{2} / \mathrm{M}$ phase were seen among cells transfected with CHFR siRNA than mock or negative control cells. On the other hand, the number of apoptotic cells significantly increased in CHFR siRNA cells after treatment with taxol. It was because knockdown of CHFR impaired the function of mitotic checkpoint, and cells lacking CHFR apparently did not stop at prophase and entered mitosis when treated with microtubule inhibitors, which triggered apoptosis. It may help to elucidate why sensitivity of taxol was enhanced in endometrial cancer cells when CHFR expression was silenced.

Our results showed that inhibition of CHFR could promote apoptosis of endometrial cancer cells when treated with taxol. However, the molecular mechanism of apoptosis promoted by CHFR suppression during mitotic stress has not yet been confirmed. Activation of cyclin B1 and its translocation to the nuclei is another important step for cells entering mitosis $(22,23)$ and precedes apoptosis induced by microtubule inhibitors $(24,25)$. In this experiment we found that nuclear localization of cyclin B1 was possibly associated with apoptosis of cells induced by CHFR suppression. Our results were consistent with several reports showing that nuclear localization of cyclin B1 correlates with sensitivity of cancer cells to chemotherapeutic drugs (7). In endometrial cancer cells during mitotic stress, apoptosis promoted by CHFR suppression, which impelled entry into mitosis without repair of DNA damage, may be related with cyclin B1 accumulation in the nuclei. However, the mechanism by which nuclear localization of cyclin B1 triggers apoptotic signaling remains to be answered in the future research.

In conclusion, CHFR can be efficiently suppressed by siRNA. Moreover, our data verified the hypothesis that suppression of CHFR expression by siRNA sensitizes endometrial cancer cells to taxol. Taken together, our study supported the application of CHFR-targeted therapies for endometrial cancer. We believe that RNA interference targeting CHFR could represent novel strategies for enhancing the sensitivity of cancer cells to taxol.

\section{Acknowledgements}

This study was supported by the grants from Endometrial Cancer Program of Guangdong Province, Science and Technology Development Fund of Macau (no. 002/2009/A), Science and Technology Plan Project of Guangdong Province (no. 2009B060700028, no. 2010B031600035) and National Natural Science Foundation of China (no. 30772332).

\section{References}

1. Okuda T, Sekizawa A, Purwosunu Y, et al: Genetics of endometrial cancers. Obstet Gynecol Int 2010: 984013, 2010.

2. Moxley KM and McMeekin DS: Endometrial carcinoma: a review of chemotherapy, drug resistance, and the search for new agents. Oncologist 15: 1026-1033, 2010.

3. Ren Y, Zhou X, Mei M, et al: MicroRNA-21 inhibitor sensitizes human glioblastoma cells U251 (PTEN-mutant) and LN229 (PTEN-wild type) to taxol. BMC Cancer 10: 27, 2010.

4. Scolnick DM and Halazonetis TD: Chfr defines a mitotic stress checkpoint that delays entry into metaphase. Nature 406: 430-435, 2000.

5. Chaturvedi P, Sudakin V, Bobiak ML, et al: Chfr regulates a mitotic stress pathway through its RING-finger domain with ubiquitin ligase activity. Cancer Res 62: 1797-1801, 2002.

6. Soutto M, Peng D, Razvi M, et al: Epigenetic and genetic silencing of CHFR in esophageal adenocarcinomas. Cancer 116: 4033-4042, 2010.

7. Ogi K, Toyota M, Mita H, et al: Small interfering RNA-induced CHFR silencing sensitizes oral squamous cell cancer cells to microtubule inhibitors. Cancer Biol Ther 4: 773-780, 2005.

8. Koga Y,Kitajima Y,Miyoshi A, Sato K, Sato S and Miyazaki K: The significance of aberrant CHFR methylation for clinical response to microtubule inhibitors in gastric cancer. J Gastroenterol 41: 133-139, 2006.

9. Wang X, Yang Y, Xu C, et al: CHFR suppression by hypermethylation sensitizes endometrial cancer cells to paclitaxel. Int J Gynecol Cancer 21: 996-1003, 2011.

10. Schmittgen TD and Livak KJ: Analyzing real-time PCR data by the comparative C(T) method. Nat Protoc 3: 1101-1108, 2008.

11. Gavet $\mathrm{O}$ and Pines J: Activation of cyclin B1-Cdk1 synchronizes events in the nucleus and the cytoplasm at mitosis. J Cell Biol 189: 247-259, 2010.

12. Porter LA, Cukier IH and Lee JM: Nuclear localization of cyclin B1 regulates DNA damage-induced apoptosis. Blood 101: 1928-1933, 2003.

13. Androic I, Kramer A, Yan R, et al: Targeting cyclin B1 inhibits proliferation and sensitizes breast cancer cells to taxol. BMC Cancer 8: 391, 2008.

14. Whitcomb BP: Gynecologic malignancies. Surg Clin North Am 88: 301-317, 2008.

15. Kim JM, Cho EN, Kwon YE, Bae SJ, Kim M and Seol JH: CHFR functions as a ubiquitin ligase for HLTF to regulate its stability and functions. Biochem Biophys Res Commun 395: 515-520, 2010.

16. Sioud M: Promises and challenges in developing RNAi as a research tool and therapy. Methods Mol Biol 703: 173-187, 2011.

17. Ashihara E: [RNA interference for cancer therapies]. Gan To Kagaku Ryoho 37: 2033-2041, 2010.

18. Qin B and Cheng K: Silencing of the IKKepsilon gene by siRNA inhibits invasiveness and growth of breast cancer cells. Breast Cancer Res 12: R74, 2010.

19. Kreutzer JN, Ruzzene M and Guerra B: Enhancing chemosensitivity to gemcitabine via RNA interference targeting the catalytic subunits of protein kinase CK2 in human pancreatic cancer cells. BMC Cancer 10: 440, 2010.

20. Kang HC, Kim IJ, Jang SG, et al: Coding region polymorphisms in the CHFR mitotic stress checkpoint gene are associated with colorectal cancer risk. Cancer Lett 260: 170-179, 2008.

21. Privette LM and Petty EM: CHFR: A novel mitotic checkpoint protein and regulator of tumorigenesis. Transl Oncol 1: 57-64, 2008.

22. Chin CF and Yeong FM: Safeguarding entry into mitosis: the antephase checkpoint. Mol Cell Biol 30: 22-32, 2010.

23. Gong D and Ferrell JE Jr: The roles of cyclin A2, B1, and B2 in early and late mitotic events. Mol Biol Cell 21: 3149-3161, 2010.

24. Heliez C, Baricault L, Barboule $\mathrm{N}$ and Valette A: Paclitaxel increases p21 synthesis and accumulation of its AKT-phosphorylated form in the cytoplasm of cancer cells. Oncogene 22: 3260-3268, 2003.

25. Summers MK, Bothos J and Halazonetis TD: The CHFR mitotic checkpoint protein delays cell cycle progression by excluding Cyclin B1 from the nucleus. Oncogene 24: 2589-2598, 2005. 